\title{
Comparison of oral, intravenous, and subcutaneous fluid therapy for resuscitation of calves with diarrhea
}

\author{
V. Doré, (i) D. M. Foster, (1) H. Ru, and G. W. Smith* (i) \\ Department of Population, Health and Pathobiology, College of Veterinary Medicine, North Carolina State University, Raleigh 27607
}

\begin{abstract}
Neonatal diarrhea remains the primary cause of mortality in dairy calves around the world, and optimal treatment protocols are needed. The main goals of therapy are to restore hydration and electrolyte concentrations, correct strong ion (metabolic) acidemia, and provide nutritional support. Administration of oral electrolyte solutions (OES) has long been the primary method used to treat neonatal diarrhea in humans and calves because OES are capable of addressing each of the primary goals of therapy. In calves with moderate dehydration, we hypothesized that oral electrolytes would be as good as or better than small volumes of intravenous (IV) or subcutaneous (SC) fluids. Therefore, the main goal of this study was to compare the ability of a commercially available oral electrolyte solution (OES) administered alone or in combination with hypertonic saline with small volumes of IV or SC fluid therapy to resuscitate calves with diarrhea. Thirty-three Holstein calves from 5 to $14 \mathrm{~d}$ of age were utilized in this clinical trial. Diarrhea and dehydration were induced by adding sucrose to the milk replacer. In addition, hydrochlorothiazide and spironolactone were given orally and furosemide intramuscularly. Depression status, clinical hydration scores, fecal consistency, and body weight were recorded at regular intervals. Treatment began when calves had severe diarrhea and had a decrease in plasma volume of at least $10 \%$. Calves were randomly assigned to 1 of 4 treatment groups of 8 to 9 calves per group: (1) OES; (2) OES with hypertonic saline (4 $\mathrm{mL} / \mathrm{kg}$, IV); (3) IV fluids (lactated Ringer's, $2 \mathrm{~L}$ ); or (4) SC fluids (lactated Ringer's, 2 L). Treatments were given at 0 and $12 \mathrm{~h}$. Changes in plasma volume, blood $\mathrm{pH}$, electrolyte levels, and physical examination scores were determined before therapy and again at 1, 2, 4, 8, and $12 \mathrm{~h}$ after each treatment. All 4 treatments were ultimately successful in improving hydration as well as increasing blood $\mathrm{pH}$; however, animals in both groups that received OES had much faster resuscitation than
\end{abstract}

Received May 15, 2019.

Accepted August 24, 2019.

*Corresponding author: Geoffrey_Smith@ncsu.edu those in either the IV or SC fluid group. In conclusion, oral electrolyte products remain the gold standard for resuscitating diarrheic calves with moderate dehydration and acidemia and will likely perform better than small volumes of IV lactated Ringer's solution. Subcutaneous fluids by themselves are a poor treatment option and should be only be used as supportive therapy following the initial correction of hypovolemia and metabolic acidosis.

Key words: dehydration, acidosis, osmotic diarrhea, oral electrolyte solution (OES), subcutaneous fluid therapy

\section{INTRODUCTION}

Neonatal diarrhea is a major source of economic loss in the dairy industry and the leading cause of preweaning calf mortality in most countries (Reiten et al., 2018; Urie et al., 2018). Treatment primarily focuses on correcting dehydration, increasing blood $\mathrm{pH}$ to correct the acidemia, restoring electrolyte concentrations and providing nutritional support (Smith and Berchtold, 2014). Several different treatments have been used to achieve these goals including oral electrolytes, intravenous (IV) fluids, and subcutaneous (SC) fluid therapy with varying results.

According to the World Health Organization, the development of oral rehydration therapy was one of the most significant advances in human medicine of 20th century. Administration of oral electrolyte solutions (OES) is often the primary method used to treat neonatal diarrhea in humans (Hartling et al., 2006; Binder et al., 2014) and calves (Smith, 2009; Trefz et al., 2012). Oral electrolyte solutions are preferred as treatment by producers because they are inexpensive, easy to administer on-farm, provide an easy way to administer large amounts of fluid, and have good efficacy for animals that still have a partially functional gastrointestinal tract. Yet, there is a large diversity of products and product quality on the market that may influence the success of electrolyte therapy, leading to confusion when evaluating treatment success (Smith, $2009,2017)$. For example, some products have very low sodium concentrations, whereas others have no alka- 
linizing agent (buffer) to correct acidemia. Therefore, some producers may have the impression that OES are not consistently effective in treating calf diarrhea because they are using a poor quality product.

In some diarrheic calves, IV fluids are used (Smith and Berchtold, 2014), and the use of SC fluid therapy remains common despite the lack of data supporting the efficacy of this route of administration in calves. Lactated Ringer's solution (LRS) is a common isotonic solution administered IV or SC in calves to correct dehydration and electrolyte abnormalities associated with neonatal diarrhea. This solution is considered a weak alkalinizing fluid because the lactate is eventually metabolized to bicarbonate in the liver, which helps increase blood $\mathrm{pH}$. However, the ability of lactate to produce an alkalinizing effect is considered weak or slow due to the metabolism requirement and it is not recommended for calves with severe acidemia (Kasari and Naylor, 1985). Although LRS has shown success in correcting dehydration and electrolyte abnormalities in calves, some disadvantages are noted. These include the requirement of IV catheter placement, special equipment for administration, animal restraint, a large amount of fluids to deliver, and the need to monitor the animal during administration (Berchtold, 2009).

The rapid delivery of hypertonic saline combined with OES administration has been shown to provide superior resuscitation compared with only OES fluid therapy (Leal et al., 2012; Aydogdu et al., 2018) or isotonic saline (Flores et al., 2006). This solution, when combined with OES, can be as effective in resuscitating severely dehydrated calves as large-volume LRS administration and is less expensive and much easier and faster to administer. Hypertonic saline is also indicated for the treatment of hyperkalemia in calves, which is commonly seen in severely dehydrated calves (Constable et al., 1996; Leal et al., 2012).

The authors have witnessed many dairy producers (particularly on large calf ranches) who routinely administer 1 to $2 \mathrm{~L}$ of LRS to calves with diarrhea either IV or SC instead of using oral electrolytes. However, it is possible that the use of oral OES (with or without IV hypertonic saline) in calves with moderate dehydration and acidosis is of equal or perhaps greater benefit than small volumes of IV or SC fluids. Therefore, the primary purpose of this study was to compare the ability of a commercially available OES alone or in combination with hypertonic saline to increase plasma volume, correct acidemia, and provide nutritional support to diarrheic calves compared with IV or SC administration of LRS. We hypothesized that OES, given with or without hypertonic saline, would resuscitate calves to the same extent as giving IV or SC LRS.

\section{MATERIALS AND METHODS}

\section{Animals}

The study protocol was approved by the North Carolina State University Institutional Committee on the Care and Use of Laboratory Animals. The study was a controlled and randomized clinical trial performed between January and October 2016. Thirty-two healthy Holstein bull calves and 1 Holstein heifer calf were used for this study. The female was born as a twin to a bull calf and was suspected to be a freemartin. Calves ranged from 35 to $56 \mathrm{~kg}$ of BW. All calves were housed in the North Carolina State University research barns for the duration of the study. Calves were acclimated to their housing and normal diet until they were between 5 and $16 \mathrm{~d}$ of age. They were fed $6 \mathrm{~L}$ of milk replacer containing 22\% protein and 20\% fat (Amplifier Max Calf Milk Replacer, Land O'Lakes Animal Milk, Arden Hills, MN) divided into 3 feeding periods (2 L per meal) per day throughout the acclimation period and for the duration of the trial. All calves were examined before induction of diarrhea for signs of clinical disease and were excluded from the study if showing signs of illness.

Calves were purchased after birth from the North Carolina State University dairy farm. Breeding heifers on this farm are vaccinated at 8 wk with clostridial (Vision 7, Merck Animal Health, Madison, NJ) and Staphylococcus aureus (Lysigin, Boehringer Ingelheim Vetmedica, Duluth, GA) bacterins along with a modified live vaccine containing infectious bovine rhinotracheitis (IBR), bovine viral diarrhea (BVD), and bovine respiratory syncytial virus (BRSV) in combination with a 5-way Leptospira bacterin (Bovishield Gold FP5, Zoetis, Parsippany, NJ). All 3 vaccines are given again at 12 wk of age. Before breeding (about 14 mo of age), heifers receive another dose of the clostridial and Staph. aureus bacterins along with a killed vaccine containing IBR, BVD, BRSV, and parainfluenza virus in combination with Leptospira (Triangle 10, Boehringer Ingelheim Vetmedica). At $7 \mathrm{mo}$ of gestation, heifers are given a gram-negative core-antigen vaccine (J-Vac, Boehringer Ingelheim Vetmedica) along with a bovine coronavirus, rotavirus, and enterotoxigenic E. coli vaccine (Scourguard $4 \mathrm{KC}$, Zoetis). Both of these vaccines are repeated 3 wk before calving. Multiparous cows receive the killed respiratory vaccine (Triangle 10), the core-antigen vaccine, and the coronavirus, rotavirus, and E. coli vaccine at the time of dry-off. Calves in this study received $3.8 \mathrm{~L}$ of colostrum after birth but did not receive any vaccinations. They were transferred to the College of Veterinary Medicine research barns within $48 \mathrm{~h}$ after birth. 


\section{Induction of Diarrhea and Dehydration}

Osmotic diarrhea and dehydration were induced by modification of a protocol that has been used extensively in previous calf diarrhea research (Constable et al., 1996; Walker et al., 1998a,b; Leal et al., 2012). Before the start of diarrhea, blood was collected to determine serum total protein concentrations. Diarrhea was induced by adding sucrose ( $3 \mathrm{~g}$ of sucrose per $\mathrm{kg}$ of BW) to the milk replacer at each feeding. In addition, calves were given a combination of diuretic drugs to promote dehydration including spironolactone at $2 \mathrm{mg} / \mathrm{kg}$ PO every $8 \mathrm{~h}$ (Patterson Vet, Greeley, CO), hydrochlorothiazide at a dose of $2 \mathrm{mg} / \mathrm{kg}$ PO every $8 \mathrm{~h}$ (Patterson Vet), and furosemide at $1 \mathrm{mg} / \mathrm{kg}$ IM every $8 \mathrm{~h}$ (Disal Injection, Boehringer Ingelheim Vetmedica). The spironolactone and hydrochlorothiazide tablets were crushed and mixed with $10 \mathrm{~mL}$ of water and given orally in 20-mL syringes. Induction of diarrhea was continued over 48 to $96 \mathrm{~h}$, depending on severity of dehydration, depression score, and fecal score. Body weight, hydration score, depression score and fecal consistency were monitored at least 3 times per day and evaluated based on the following (0 to 3) scale. Skin tent examinations were performed in the neck.

- Hydration score: $0=$ normal (eyes are bright and not recessed into orbit, skin feels pliable); $1=$ mild dehydration (slight loss of skin elasticity, skin tent duration $2-3 \mathrm{~s}$, eyes normal to mildly recessed into orbit); $2=$ moderate dehydration (skin tent $>3 \mathrm{~s}$ but $<10 \mathrm{~s}$, eyes moderately recessed into orbit); 3 $=$ severe dehydration (skin tent $>10 \mathrm{~s}$, eyes markedly recessed into orbit).

- Depression scoring: $0=$ normal; $1=$ mild depression (calf suckles but not vigorously); 2 = moderate depression (calf able to stand, suckle is weak or disorganized); $3=$ severe depression (calf unable to stand or suckle).

- Fecal consistency: 0 = normal (manure is normal and well formed); $1=$ abnormal feces but not diarrhea (pasty manure, softer than normal); 2 $=$ mild diarrhea (semi-liquid but still has a solid component); $3=$ severe diarrhea (pure liquid, splashes when it hits the ground and does not form any solid structure).

\section{Experimental Protocol}

This model reliably induces diarrhea and moderate to severe dehydration within 48 to $72 \mathrm{~h}$. Therefore, physical examinations were done every $8 \mathrm{~h}$ until calves had achieved moderate dehydration (hydration score of 2 and plasma volume decrease of at least 10\%), a depression score of at least 1, and a fecal consistency score of 3. Before starting treatment, each calf had a jugular catheter placed to facilitate blood sampling and fluid administration. When calves qualified to enter the treatment phase of the study, they were assigned to 1 of the following 4 groups using a random number generator: (1) Group 1 ( $\mathrm{n}=9$ calves), oral electrolytes: calves received a packet of OES (Diaque, Boehringer Ingelheim Vetmedica) mixed with water according to label directions (2 quarts or 1,891 mL of water). Calves were allowed to suckle the OES but were fed using an esophageal feeder when necessary. Calves weighed 46.5 $\pm 5.1 \mathrm{~kg}$ (mean $\pm \mathrm{SD}$ ) and were $10.6 \pm 2.8 \mathrm{~d}$ of age at the time of diarrhea induction. (2) Group $2(\mathrm{n}=8$ calves), IV fluids: calves received 2 L of LRS through a jugular catheter over a 30 -min period. Calves in this group weighed $47.1 \pm 4.8 \mathrm{~kg}$ (mean $\pm \mathrm{SD}$ ) and were $10.2 \pm 2.6 \mathrm{~d}$ of age at the time of diarrhea induction. (3) Group 3 ( $\mathrm{n}=8$ calves), SC fluids: calves received 2 L of LRS given subcutaneously. The fluids were administered over 10 to $15 \mathrm{~min}$ in 4 different sites, 500 $\mathrm{mL}$ per site, over the thorax and neck. Calves in this group weighed $47.1 \pm 4.2 \mathrm{~kg}$ (mean $\pm \mathrm{SD}$ ) and were $10.5 \pm 3.3 \mathrm{~d}$ of age at the time of diarrhea induction. (4) Group 4 ( $n=8$ calves), oral electrolytes and hypertonic saline (OES+HSS): calves received an infusion of $7.2 \%$ hypertonic saline through a jugular catheter at a dose of $4 \mathrm{~mL}$ per $\mathrm{kg}$ of BW over 5 min. This was immediately followed by a feeding of OES mixed in water according to label directions (2 quarts or 1,891 $\mathrm{mL}$ of water). Calves weighed $46.6 \pm 4.1 \mathrm{~kg}$ (mean \pm $\mathrm{SD}$ ) and were $9.6 \pm 3.7 \mathrm{~d}$ of age at the time of diarrhea induction.

The composition of the oral electrolyte product used in this study is compared with LRS and hypertonic saline in Table 1. At the time of enrollment into treatment groups, all administration of diuretics was stopped. Blood was collected at baseline (before treatment or time 0 ) and then at $1,2,4,8$, and $12 \mathrm{~h}$ after treatment to determine change in plasma volume, electrolyte concentrations, and blood gas analysis. Using a portable blood chemistry analyzer (i-Stat 1, Abaxis North America, Union City, CA), the following were measured at each time point: blood $\mathrm{pH}$, partial pressure of $\mathrm{CO}_{2}\left(\mathrm{pCO}_{2}\right)$, bicarbonate, base excess, hematocrit, hemoglobin, glucose, sodium, chloride, potassium, and anion gap concentrations. The anion gap (AG), which is defined as the difference between unmeasured cations and unmeasured anions, was calculated in milliequivalents per liter as follows: $\left(\left[\mathrm{Na}^{+}\right]+[\mathrm{K}+]-\left(\left[\mathrm{Cl}^{-}\right]\right.\right.$ $\left.+\left[\mathrm{HCO}_{3}^{-}\right]\right)$.

In addition, serum protein was determined by a chemistry analyzer (Cobas c 501, Roche Diagnostics, Indianapolis, IN). Actual blood gas values were used in 
Table 1. Sodium, chloride, potassium, buffer, osmolality, and strong ion difference (SID) values of the various treatments used in this study

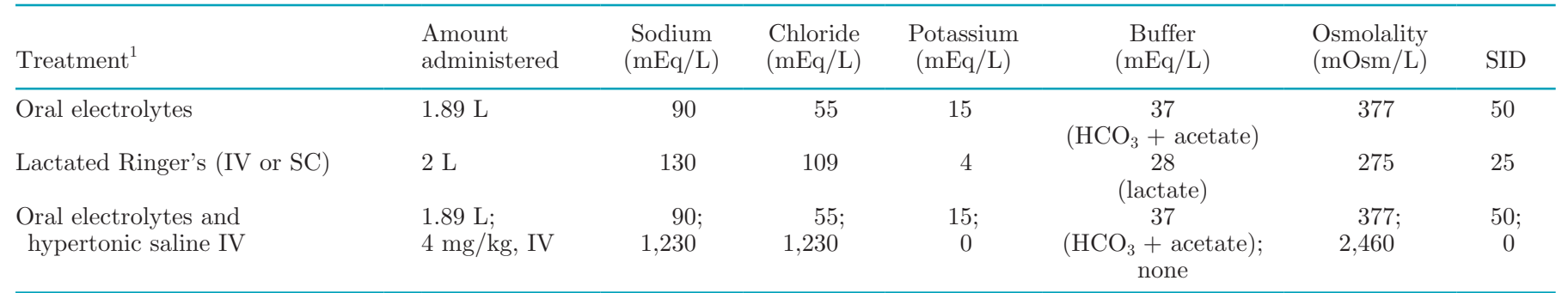

${ }^{1} \mathrm{IV}=$ intraveneous; $\mathrm{SC}=$ subcutaneous.

the data analysis (not corrected for rectal temperature). Bicarbonate values were calculated using the formula $\log \mathrm{HCO}_{3}{ }^{-}=\mathrm{pH}+\log \mathrm{pCO}_{2}-7.608$; and extracellular base excess values $=\mathrm{HCO}_{3}^{-}-24.8+16.2(\mathrm{pH}-7.4)$. The change in plasma volume at each time point was determined by the following formula:

$$
\left(\mathrm{Pt}_{0}-\mathrm{Pt}_{\mathrm{x}}\right) \times 100 / \mathrm{Pt}_{\mathrm{x}}
$$

where $\mathrm{Pt}_{0}$ is the serum protein concentration before induction and $\mathrm{Pt}_{\mathrm{x}}$ is the serum protein concentration at each measured time point after treatment.

Approximately $12 \mathrm{~h}$ after initial therapy, calves received a second fluid administration within their assigned groups; that is, group 1 again received OES, group 2 received another $2 \mathrm{~L}$ of LRS IV, and so on. Blood was collected before the second treatment as well as $1,2,4,8$, and $12 \mathrm{~h}$ after the second treatment to assess further changes of fluid therapy on plasma volume, acid-base status, and electrolyte concentrations. After the last data were collected, calves were humanely euthanized with an overdose of pentobarbital (Euthasol, Virbac, Fort Worth, TX) intravenously through their catheter.

\section{Statistical Analysis}

Data were assessed for a normal distribution (Proc Univariate in SAS version 9.4; SAS Institute Inc., Cary, $\mathrm{NC}$ ), and the outcomes for continuous variables were modeled using repeated-measures ANOVA (Proc Mixed in SAS 9.4), where treatment, time, and their interaction were used as factors. The covariance structure among the repeated measures was modeled via autoregressive model. Least squares means were estimated and compared between treatments by time between treatments overall and between time points within each treatment. Adjusted $P$-values (Tukey method) are presented.

The ordinal response was modeled using proportional odds model (Proc Genmod in SAS 9.4), where treatment, time, and their interaction were used as explanatory variables. The covariance structure among the repeated measures was modeled via the independent working correlation matrix. Probabilities were estimated and compared between treatments by time, between treatments overall, and between time points within each treatment. Adjusted $P$-values (Tukey method) are presented.

\section{RESULTS}

\section{Response to Induction of Diarrhea}

Most calves met the criteria for inclusion in the study between 40 and $72 \mathrm{~h}$, with the largest majority between 64 and $72 \mathrm{~h}(\mathrm{n}=20)$. Two calves required 96 $\mathrm{h}$ after induction before they met the criteria to begin treatment. All calves developed moderate metabolic acidosis (mean \pm SD: $7.24 \pm 0.04$; range: 7.09 to 7.30 ) and showed clinical signs of dehydration including prolonged skin tent $(>3 \mathrm{~s})$, enophthalmos $(\geq 3 \mathrm{~mm})$, and loss of at least $10 \%$ of their BW. Calves maintained a weak to good suckle reflex throughout the study, and only one calf in the OES group had to be fed using an esophageal feeder at the first treatment. Calves also continued drinking their normal milk allotment $(2 \mathrm{~L})$ through the study period.

All calves presented with fecal, hydration, and attitude scores of 0 on d 1 (before the start of diarrhea induction). Response to the osmotic diarrhea protocol was noted almost immediately in most calves. A fecal score of 3 was seen in almost all calves $(\mathrm{n}=31) 8 \mathrm{~h}$ following induction and in all calves at $24 \mathrm{~h}$ following induction (Table 2). Fecal score at the end of the study was around 1 and decreased rapidly after removal of sucrose in the milk, which occurred $16 \mathrm{~h}$ after the initial treatment. Hydration status was at least 2 in all calves at the beginning of the treatment period, with 2 calves having a score of 3 . At the end of the study, the hydration score was back to 0 except in the IV group, but this was not significantly different. Highest median hydration score for all groups occurred at the beginning of the treatment period until $8 \mathrm{~h}$ post-treatment. Attitude score was variable at the beginning of the 
Table 2. Median attitude, hydration, and fecal scores following first and second treatments in calves with osmotic diarrhea ${ }^{1}$

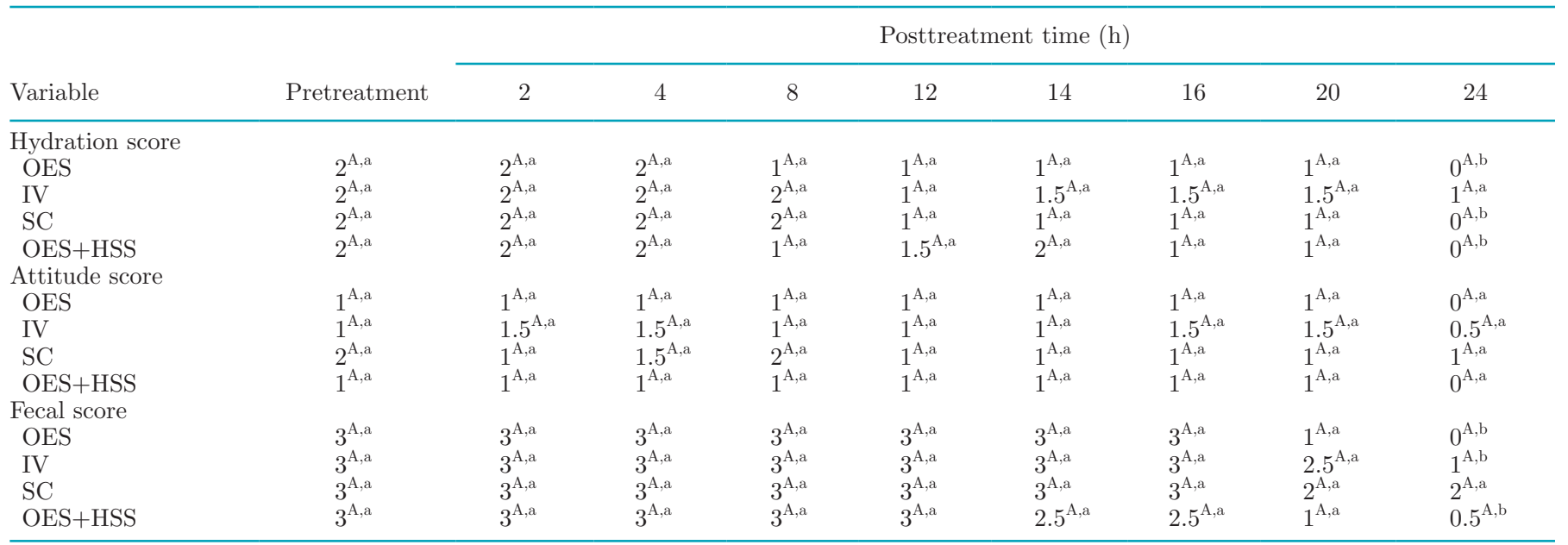

${ }^{\mathrm{A}}$ Values with the same letter are not significantly different from each other between treatment groups.

${ }^{\mathrm{a}, \mathrm{b}}$ Values with different letters are significantly different between experimental time points for the same treatment $(P<0.05)$.

${ }^{1} \mathrm{OES}=$ oral electrolyte solution; IV = intravenous fluids (lactated Ringer's); SC = subcutaneous fluids (lactated Ringer's); OES+HSS = oral electrolytes and hypertonic saline $(7.2 \%)$.

treatment period with 20 calves having a score of 1,12 having a score of 2 , and 1 having a score of 3 . Median attitude score for all groups was around 1 for the entire treatment period. Severe plasma volume depletion was seen in all groups when comparing plasma protein concentrations before induction with plasma protein levels at the beginning of the treatment period. The $\mathrm{BW}$ decrease from the time of diarrhea induction until the beginning of treatment was $15.4 \%$ across groups. Calves weighed $46.6 \pm 5.2 \mathrm{~kg}$ (mean $\pm \mathrm{SD}$ ) before the induction of diarrhea and $39.3 \pm 4.3 \mathrm{~kg}$ at the time of initial treatment, with no difference between groups and $46.1 \pm 5.3 \mathrm{~kg}$ at the conclusion of the study.

\section{Effect of Treatment}

Changes in total protein, AG, sodium, chloride, potassium, hematocrit, and bicarbonate concentrations are summarized in Table 3. All calves presented with an increase in plasma protein concentration compared with pre-induction values at the time of first treatment. The fastest decrease in total protein was noted in the IV group, with a significant difference at $1 \mathrm{~h}$ posttreatment. The SC group showed a slower decrease in plasma protein compared with the other 3 groups.

Both the IV and OES+HSS groups had faster and more significant improvements in plasma volume beginning $1 \mathrm{~h}$ following each treatment, with the OES group showing a similar improvement $2 \mathrm{~h}$ following treatment (Figure 1). The SC fluid group was the slowest to show increases in plasma volume, being statistically lower until $8 \mathrm{~h}$ after the first treatment and $4 \mathrm{~h}$ following the second treatment. Hematocrit was also slower to decrease in the SC group compared with all other groups.

The OES group had an increase in blood $\mathrm{pH}$, bicarbonate, and base excess starting $8 \mathrm{~h}$ following initial treatment (Figures 2 and 3). Glucose levels were significantly affected by the inclusion of OES (Figure 4) for the first $2 \mathrm{~h}$ following each treatment. As expected, significant differences were observed in sodium concentration following infusion with hypertonic saline. Interestingly, hypertonic saline did not significantly increase chloride levels. Potassium was the only value not affected by treatment. Treatment group had no significant effect on weight, attitude, hydration, or fecal score.

\section{Effect of Time}

In the OES group, significant findings were noted for glucose, potassium, chloride, blood $\mathrm{pH}$, plasma volume, sodium, and total protein. Glucose was significantly increased 1 and $2 \mathrm{~h}$ following each treatment. Potassium levels decreased significantly $1 \mathrm{~h}$ following treatment, with a significant effect following the second treatment. Chloride and sodium both showed a progressive decrease, with significantly lower concentrations at 20 and $24 \mathrm{~h}$. Plasma volume showed a rapid increase $1 \mathrm{~h}$ following OES ingestion, and blood $\mathrm{pH}$ increased starting $1 \mathrm{~h}$ following the first treatment and continued to improve over the course of the treatment period. Intravenous fluids were also effective in increasing plasma volume and decreasing plasma protein levels as soon as $1 \mathrm{~h}$ after treatment. 


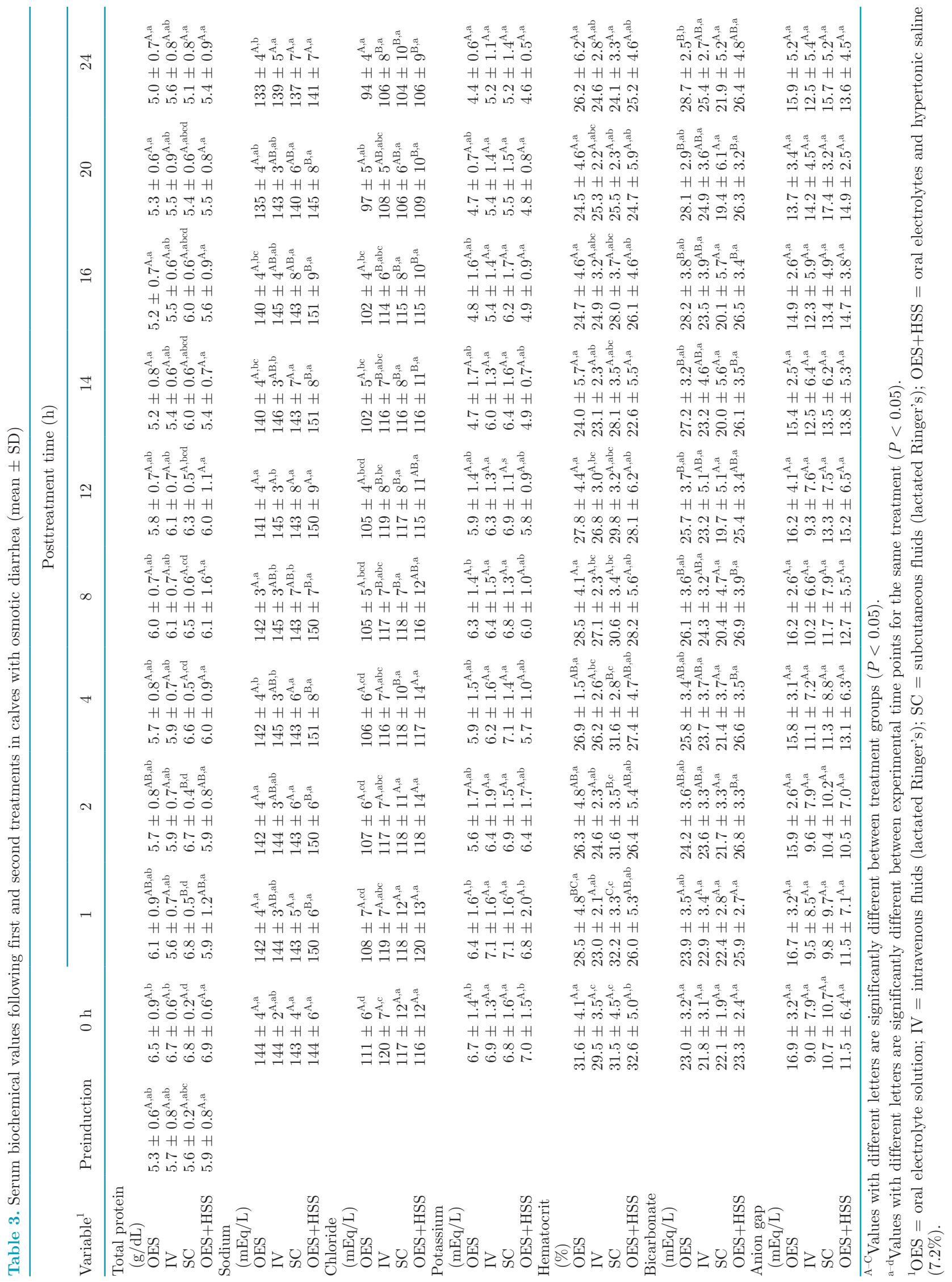




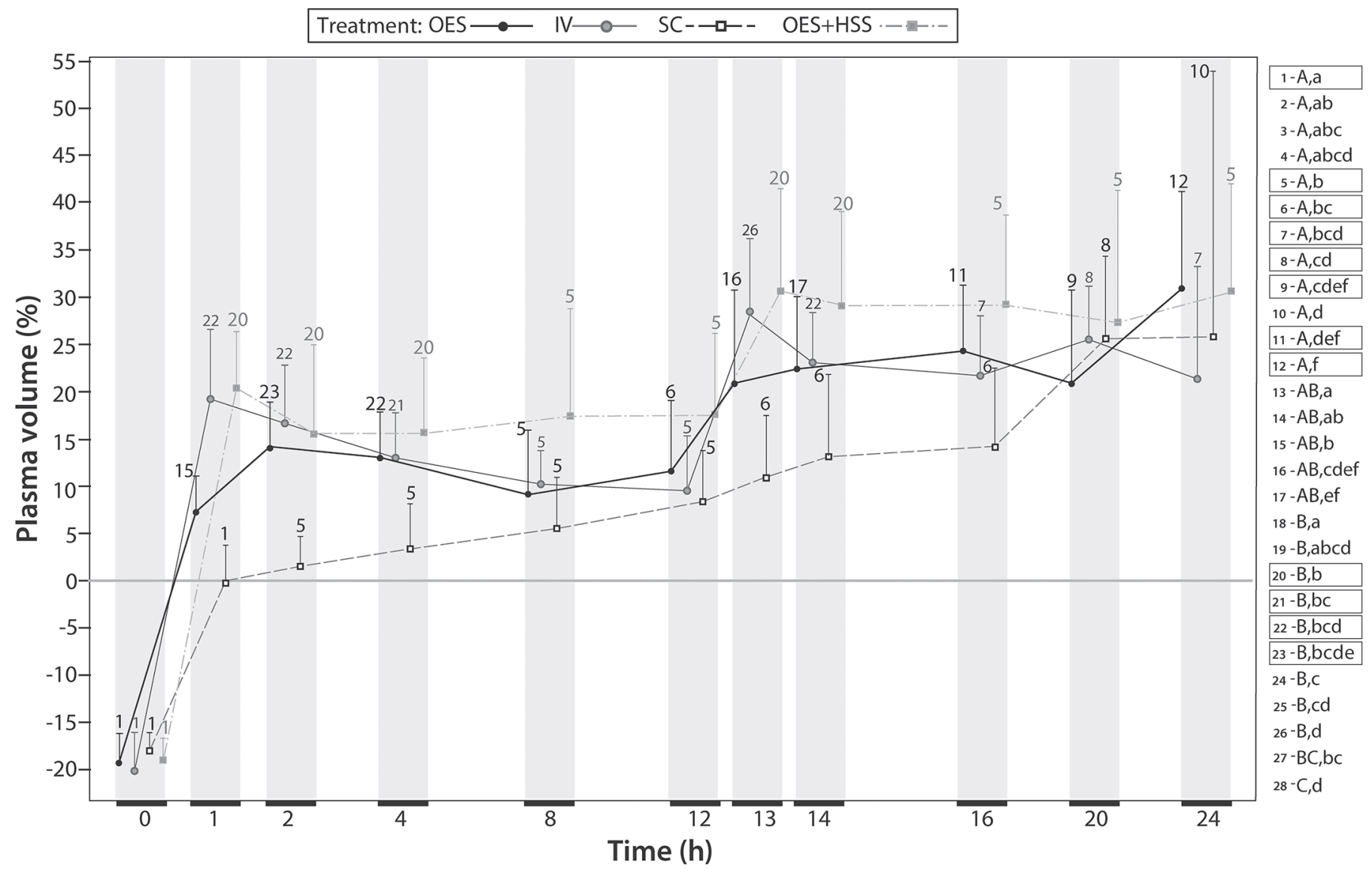

Figure 1. Change in plasma volume following first and second treatments in calves with osmotic diarrhea. Treatment OES $=$ oral electrolytes $(\mathrm{n}=9) ; \mathrm{IV}=$ intravenous fluids $(\mathrm{n}=8) ; \mathrm{SC}=$ subcutaneous fluids $(\mathrm{n}=8) ;$ OES + HSS $=$ oral electrolytes and hypertonic saline $(7.2 \%)(\mathrm{n}=$ 8). Data points with different uppercase letters (A-C) differ significantly between treatment groups; data points with different lowercase letters $(\mathrm{a}-\mathrm{f})$ differ significantly between experimental time points $(P<0.05)$. Error bars indicate SE.

The SC treatment show a decrease in hematocrit and total protein, with an increase in plasma volume over time; however, the improvements were much slower to occur than in the other groups. The effects of SC fluid therapy on blood $\mathrm{pH}$, base excess, and bicarbonate were not significant from one period to another.

Similar to IV fluids, the OES+HSS treatment was particularly effective in increasing plasma volume and decreasing plasma protein levels as soon as $1 \mathrm{~h}$ following infusion. Similar to the OES group, the OES+HSS group showed a rapid reduction in potassium levels beginning $1 \mathrm{~h}$ after infusion. Glucose levels also increased significantly 1 and $2 \mathrm{~h}$ after both treatments.

\section{DISCUSSION}

The most significant finding of this study was that OES, with or without hypertonic saline, was superior to $2 \mathrm{~L}$ of LRS given by the IV or SC routes for resuscitating calves with diarrhea. The improvement in plasma volume seen with OES alone was almost as rapid as that achieved with small volumes of IV fluids, and the improvement in blood $\mathrm{pH}$ and glucose concentrations was much faster. As shown previously (Constable et al., 1996; Leal et al., 2012; Aydogdu et al., 2018), hypertonic saline was effective in increasing the ability of OES to improve hydration status in dehydrated calves. In contrast, subcutaneous fluid administration was very slow to increase plasma volume or blood $\mathrm{pH}$ in this study and likely represents a poor option in calves for treating diarrhea.

The oral electrolyte solution used in this study was a mildly hypertonic alkalinizing OES containing lecithincoated citrus fiber. This efficacy of this OES has been demonstrated previously, with superior performance compared with other electrolyte products (Constable et al., 2009; Bachmann et al., 2012; Goodell et al., 2012). Because it does not interfere with milk clotting and has moderate osmolality, it has the advantage of having label directions that allow mixing with milk to provide better energy supplementation and weight gain (Goodell et al., 2012). As was seen in previous studies, 


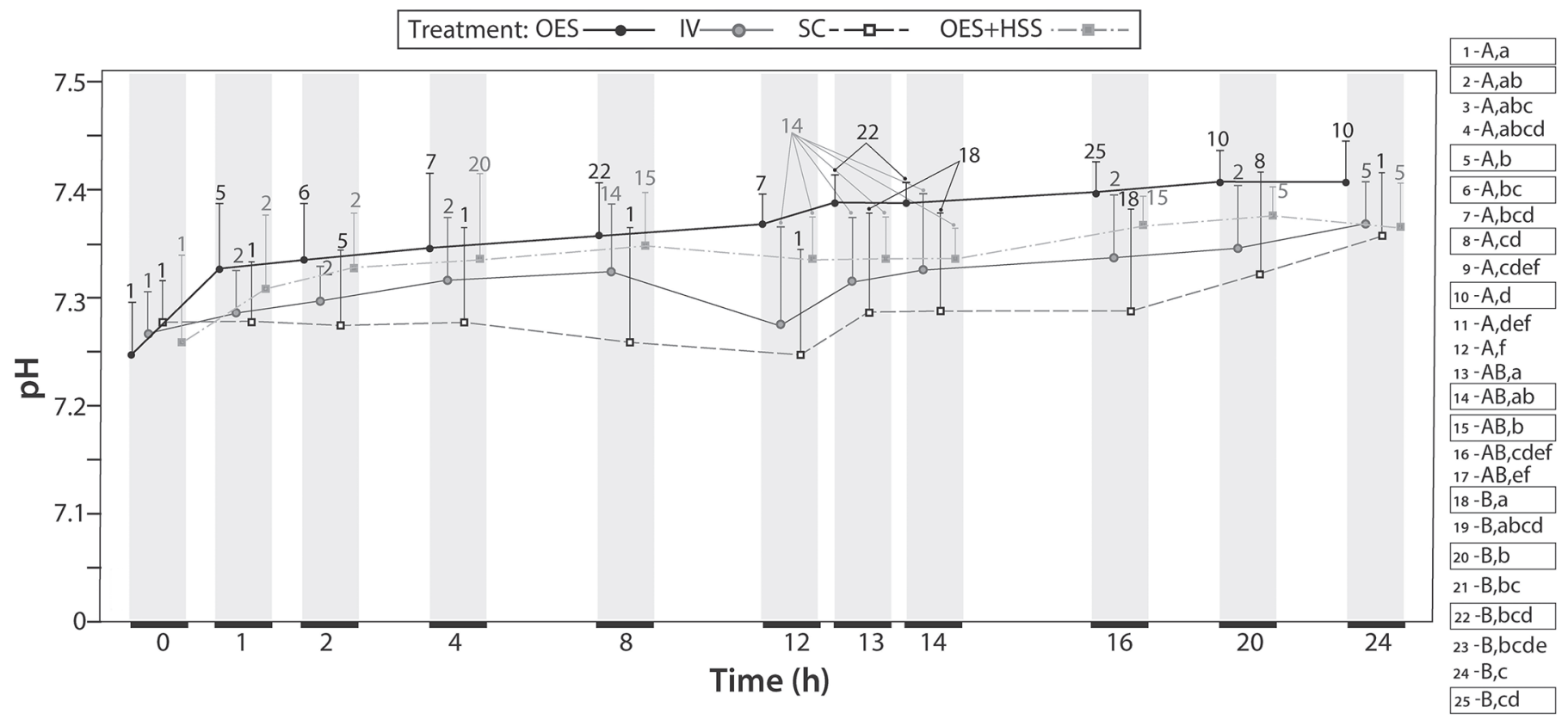

Figure 2. Change in blood $\mathrm{pH}$ following first and second treatments in calves with osmotic diarrhea. Treatment OES $=$ oral electrolytes $(\mathrm{n}$ $=9) ; \mathrm{IV}=$ intravenous fluids $(\mathrm{n}=8) ; \mathrm{SC}=$ subcutaneous fluids $(\mathrm{n}=8) ; \mathrm{OES}+\mathrm{HSS}=$ oral electrolytes and hypertonic saline $(7.2 \%)(\mathrm{n}=8)$. Data points with different uppercase letters (A, B) differ significantly between treatment groups; data points with different lowercase letters $(\mathrm{a}-\mathrm{f})$ differ significantly between experimental time points $(P<0.05)$. Error bars indicate SE.

we found that this OES demonstrated a good capacity to improve plasma volume, replace electrolytes, treat moderate metabolic acidosis, and support blood glucose concentrations.

One of the goals in formulating an OES is to optimize the glucose-sodium cotransporter at the level of the enterocytes (Binder et al., 2014). Oral electrolyte solutions that are well designed to optimize the use of this cotransporter will allow better absorption of electrolytes and glucose. A negative energy balance and loss of BW are common findings in calves with diarrhea because of a reduction in energy intake and decreased absorption of nutrients by the gut. Severe hypoglycemia has also been associated with an increased risk of mortality in calves with diarrhea (Trefz et al., 2016, 2017). A significant advantage of using the OES noted in this study was the increase in blood glucose levels following treatment, thus providing immediate energy. This finding supports the use of OES with or without IV fluid to help supplement glucose and energy to the diarrheic calf.

It should be noted that the $2 \mathrm{~L}$ of LRS administered intravenously in this study was less than the volume required to replace the fluid deficit in these calves. For example, calves weighed about $40 \mathrm{~kg}$ in this study at the time of initial treatment and had roughly a $19 \%$ decrease in plasma volume. Therefore, these calves would need at least $7.5 \mathrm{~L}(40 \times 0.19)$ of fluids just to correct their deficit (not accounting for ongoing losses from continued diarrhea). If we had given a greater volume of LRS IV, we might have seen better results. However, we elected to give $2 \mathrm{~L}$ of IV fluids to more closely mimic what is done in most field scenarios, although previous studies comparing higher volumes of IV LRS with OES+HSS have shown similar results. In one study, a group of calves were given $80 \mathrm{~mL} / \mathrm{kg}$ of LRS for the first hour followed by $4 \mathrm{~mL} / \mathrm{kg}$ per hour for an additional 7 h (Walker et al., 1998b). For a 40-kg calf, this would be approximately $5.5 \mathrm{~L}$ of IV LRS given over an 8-h period. Calves treated with OES+HSS in this study had more significant increases in plasma volume that were sustained for a significantly longer period compared with the group receiving the 8 -h infusion of LRS. A recent study gave IV LRS at the rate of $30 \mathrm{~mL} / \mathrm{kg}$ per hour for $3 \mathrm{~h}$ (about 3.6 L total for a 40-kg calf) to a group of calves with naturally occurring diarrhea (Aydogdu et al., 2018). A second group of calves received the same rate of acetated Ringer's and a third group received 4 $\mathrm{mL} / \mathrm{kg}$ of OES $+\mathrm{HSS}$. Significant increases in plasma volume were noted in the OES+HSS group within 30 min of treatment but not in the other 2 groups. As seen in the study by Walker et al. (1998b), the OES+HSS group had a more significant and sustained increase in plasma volume than either group that received IV fluids. Because of different sampling times in this study, we were not able to see the increase in plasma volume 
at 30 min post-infusion; however, we noticed a similar effect on plasma volume in the OES+HSS and IV fluid groups at $1 \mathrm{~h}$ post-treatment ( 1 and $13 \mathrm{~h}$ ).

Despite the relatively common use of SC fluids in calves with diarrhea, this is the first study to include data on their use in calves. In small animal species, SC fluids are commonly used in mildly dehydrated patients (Smith and Greer, 2016) or as supportive therapy in cases of chronic renal disease (Cooley et al., 2018). Recommendations are to not use SC fluids in hypovolemic patients because of the limited absorption associated with peripheral vasoconstriction. As in other species, dehydration in calves in this study showed slow and progressive improvement after SC fluid therapy. However, the effects on acid-base status and electrolyte concentrations were minimal until 16 to $20 \mathrm{~h}$ into the study period. The data from this study indicate that small volumes of SC fluids should not be used as the sole treatment for rapid correction of dehydration and metabolic acidosis in calves with diarrhea.
The osmotic diarrhea model utilized in this study was modified slightly from previously published models. The original model included adding sucrose to the milk replacer at $2 \mathrm{~g}$ per $\mathrm{kg}$ of $\mathrm{BW}$ along with the use of furosemide $(2 \mathrm{mg} / \mathrm{kg}$, IM, every $6 \mathrm{~h})$, spironolactone (1 $\mathrm{mg} / \mathrm{kg}, \mathrm{PO}$, every $8 \mathrm{~h}$ ), and hydrochlorothiazide (1 $\mathrm{mg} / \mathrm{kg}, \mathrm{PO}$, every $8 \mathrm{~h}$ ). This model is extremely effective in inducing severe diarrhea (Walker et al., 1998a) and has been used in multiple studies evaluating fluid resuscitation in calves with diarrhea (Constable et al., 1996; Walker et al., 1998b). Although this model is very effective in producing profound dehydration, calves do not typically develop a significant metabolic acidosis. A more recent study increased the dose of sucrose to $4 \mathrm{mg} / \mathrm{kg}$, increased the dose of both spironolactone $(2$ $\mathrm{mg} / \mathrm{kg}, \mathrm{PO}$, every $8 \mathrm{~h}$ ) and hydrochlorothiazide (2 mg/ $\mathrm{kg}$, PO, every $8 \mathrm{~h}$ ), but eliminated the use of furosemide (Leal et al., 2012). The newer model was effective in producing dehydration but calves also had moderate acidosis at the time treatment was started (blood $\mathrm{pH}$

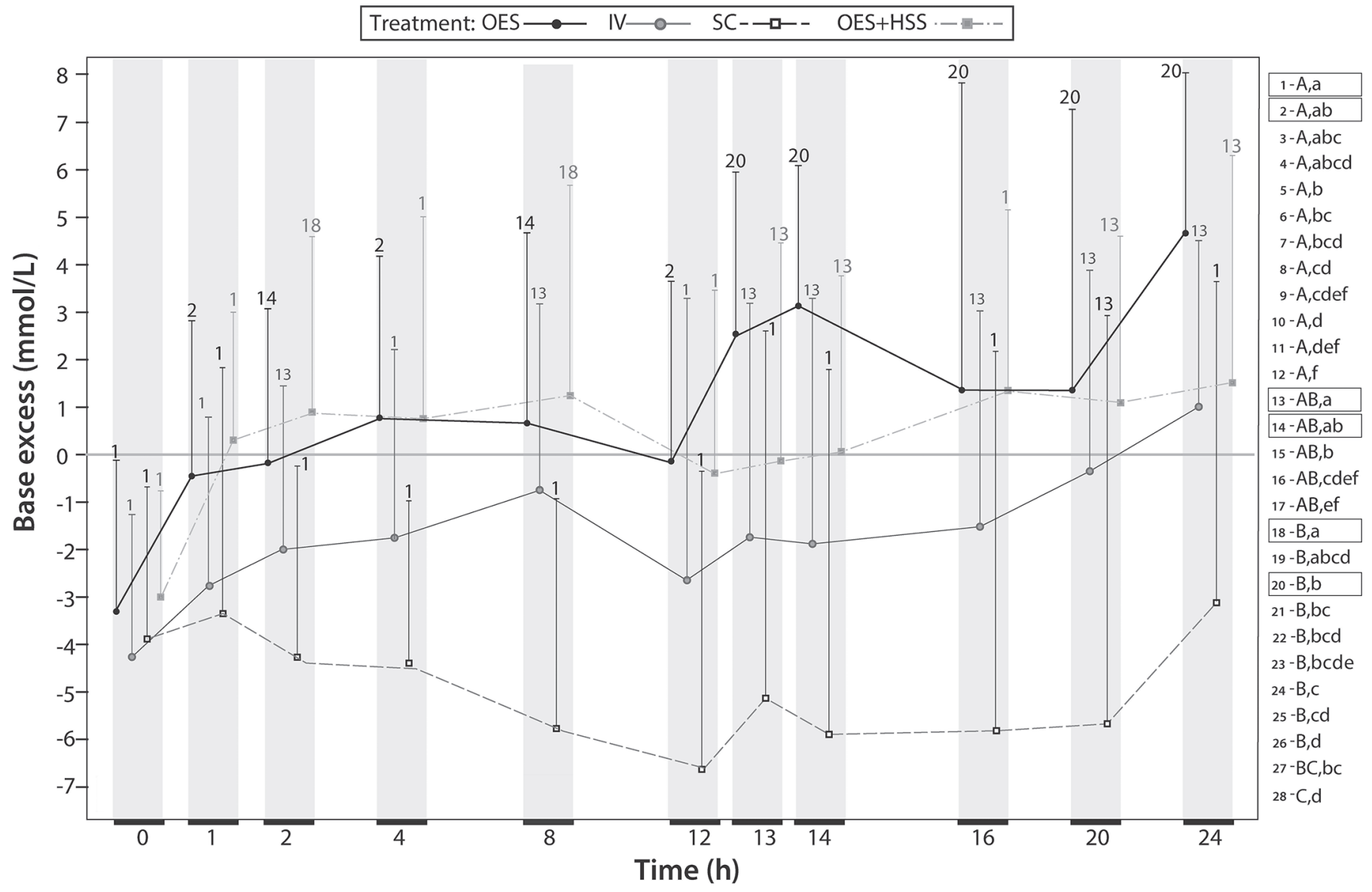

Figure 3. Change in base excess concentration following first and second treatments in calves with osmotic diarrhea. Treatment OES $=$ oral electrolytes $(\mathrm{n}=9) ; \mathrm{IV}=$ intravenous fluids $(\mathrm{n}=8) ; \mathrm{SC}=$ subcutaneous fluids $(\mathrm{n}=8)$; OES+HSS = oral electrolytes and hypertonic saline $(7.2 \%)(\mathrm{n}=8)$. Data points with different uppercase letters $(\mathrm{A}-\mathrm{C})$ differ significantly between treatment groups; data points with different lowercase letters $(\mathrm{a}-\mathrm{f})$ differ significantly between experimental time points $(P<0.05)$. Error bars indicate SE. 


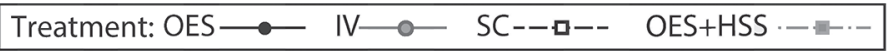

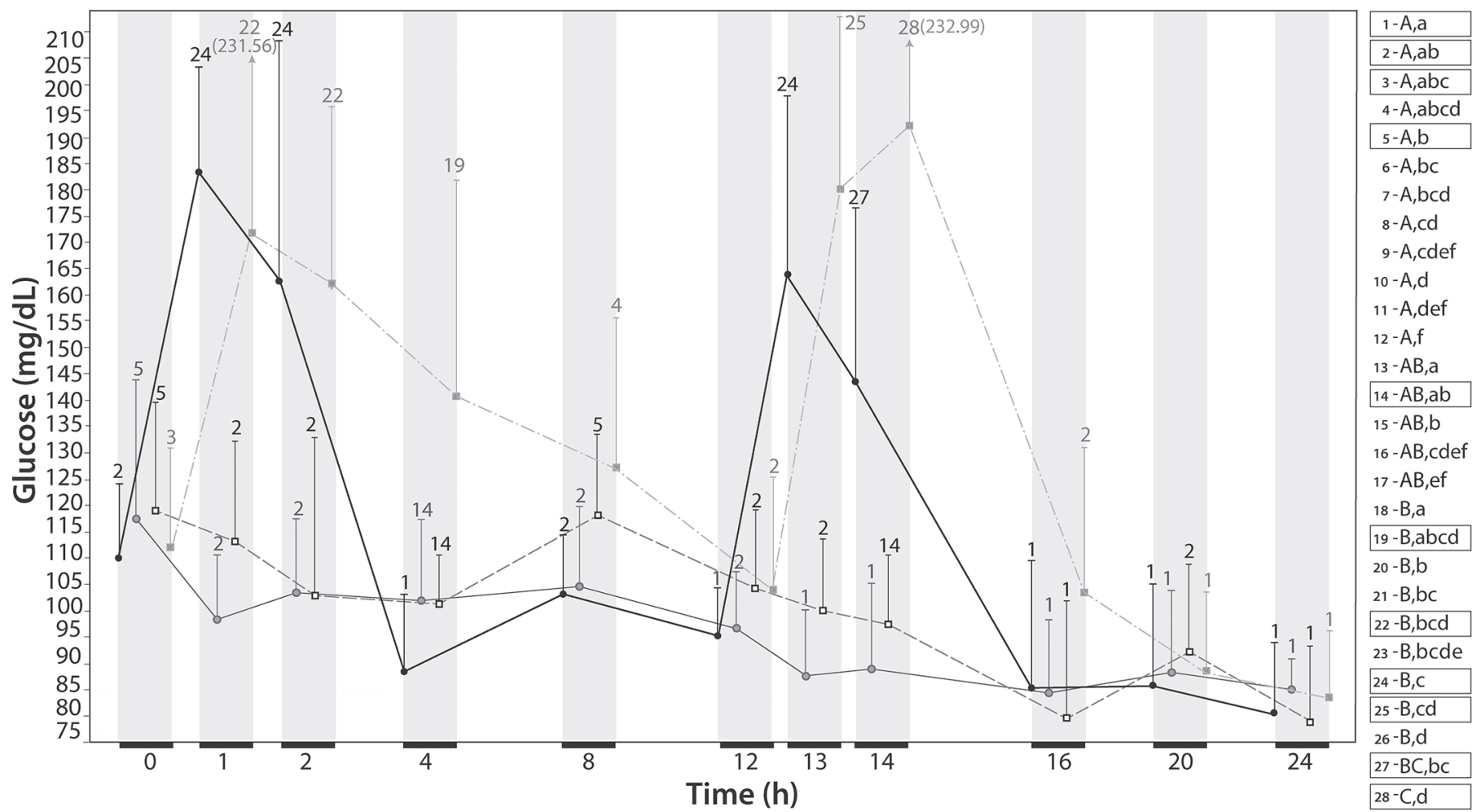

Figure 4. Change in blood glucose following first and second treatments in calves with osmotic diarrhea. Treatment OES $=$ oral electrolytes $(\mathrm{n}=9) ; \mathrm{IV}=$ intravenous fluids $(\mathrm{n}=8) ; \mathrm{SC}=$ subcutaneous fluids $(\mathrm{n}=8) ;$ OES+HSS $=$ oral electrolytes and hypertonic saline $(7.2 \%)(\mathrm{n}=$ 8). Data points with different uppercase letters $(\mathrm{A}-\mathrm{C})$ differ significantly between treatment groups; data points with different lowercase letters $(\mathrm{a}-\mathrm{f})$ differ significantly between experimental time points $(P<0.05)$. For data points where the standard error margin exceeds the maximum boundary of the y-axis, the values are indicated numerically.

7.23 to 7.27$)$. We originally began with that model but the first group of calves had very high blood sodium concentrations (175 to $189 \mathrm{mEq} / \mathrm{L}$ ) along with clinical signs of hypernatremia. Ultimately, these calves had to be removed from the study, and we elected to decrease the sucrose dose to avoid high sodium levels. The previous study (Leal et al., 2012) fed calves whole milk, whereas we used milk replacer in this study. Whole milk is typically low in sodium, averaging about 17 to $28 \mathrm{mmol} / \mathrm{L}$, although this may increase to 35 to $45 \mathrm{mmol} / \mathrm{L}$ in late gestation or in cows with mastitis (Gaucheron, 2005). In contrast, many milk replacer products have significantly higher sodium concentrations than whole milk. The sodium concentration of the milk replacer used in this study (as mixed) was $88 \mathrm{mEq} / \mathrm{L}$, likely making calves more susceptible to hypernatremia than those in the study by Leal et al. (2012). Sucrose doses of $4 \mathrm{mg} / \mathrm{kg}$ can be used in future studies; however, it is likely necessary to use whole milk or milk replacers with lower sodium levels to avoid salt toxicity. However, by lowering the sucrose dose slightly and adding lower doses of furosemide, we were able to produce a model of osmotic diarrhea that still produced moderate acidosis.

Serum potassium levels at the time of initial treatment were elevated above the normal range in all calves in this study. In one study looking at 124 calves with diarrhea, $34 \%$ had hyperkalemia (serum potassium concentration $>5.8 \mathrm{mEq} / \mathrm{L}$ ) at the time of initial treatment (Trefz et al., 2013a). In a larger study of 832 calves presenting to a university teaching hospital for diarrhea, $28 \%$ had elevated serum potassium levels (Trefz et al., 2013b). In the study by Trefz et al. (2013b), hyperkalemia was primarily associated with dehydration and not necessarily whether the calves had an acidemia or not. The increase in potassium concentrations in our study was likely not due to furosemide administration, because this drug should increase potassium excretion by the nephron. Furosemide is a loop diuretic and is known to act on the $\mathrm{Na}-\mathrm{K}-2 \mathrm{Cl}$ transport protein to reduce reabsorption of sodium, potassium, and chloride (Roush et al., 2014). Trefz and Lorenz (2017) concluded that hyperkalemia is highly dependent on the degree of dehydration, which acts directly on aldosterone- 
induced potassium control mechanisms. According to their findings, even if aldosterone is markedly elevated in hyperkalemic calves with diarrhea, renal excretion of potassium is not sufficient to counterbalance the systemic increase caused by severe hypovolemia. In addition, elevated levels of D-lactate may also help prevent hyperkalemia (Trefz et al., 2013b). Although D-lactate concentrations were not measured in this study, they were likely not significantly elevated because the acidosis seen in this study was not severe. The hypovolemia observed in this study likely explains the higher levels of potassium seen at the time of initial treatment. The elevated potassium concentrations decreased with all treatments over time but decreased the fastest (but not significantly faster) with OES or OES+HSS.

A potential limitation of this study is that it used an experimental model of osmotic diarrhea instead of calves with naturally occurring diarrhea. Although this protocol has been used extensively in previous studies to examine different approaches for fluid resuscitation in diarrheic calves (Constable et al., 1996; Walker et al., 1998a,b; Leal et al., 2012), it could be argued that it does not reproduce the exact metabolic alterations that are seen in some calves with diarrhea. In many acquired cases of neonatal diarrhea, calves have hyponatremia, elevated levels of D-lactate, and an elevated AG (Trefz et al., 2015). Although D-lactate was not measured in this study, sodium levels were within the normal range and calves had a relatively mild acidemia overall compared with most calves with severe dehydration in the field. Therefore, this model might induce a more significant dehydration than it does severity of acidemia. However, previous studies have demonstrated no relationship between the severity of acidosis and the degree of dehydration (Naylor, 1987; Grove-White and White, 1993). It should be emphasized, however, that calves with more severe acidosis need IV sodium bicarbonate (Trefz et al., 2012; Smith and Berchtold, 2014). Clinically, this is often defined as a blood pH of $<7.20$ or a calf that is unable to stand during physical examination. These calves are unlikely to benefit solely from OES.

Another potential limitation is that the 4 treatments used in this study were not equal. As outlined in Table 1, they have different sodium, chloride, potassium, glucose, and buffer concentrations along with varying strong ion differences and osmolalities. Although the treatments varied, they were chosen to represent common treatments given to calves with diarrhea in North America. The primary purpose of this study was to compare 4 very commonly administered treatments that are used in the field, not to ensure each treatment was equivalent in terms of sodium concentration, and so on. Certainly this approach leads to limitations in terms of interpreting the data. For example, both groups of calves that received OES had much greater increases in plasma glucose concentrations than calves that received small volumes of IV or SC LRS. However, if additional dextrose had been added to the LRS solutions, the results would have likely been very different. Therefore, it should not be assumed that OES is always superior to IV fluid therapy; our conclusions should only be extended to the treatment groups utilized in this study (small volumes of LRS given IV or SC).

As long as diarrhea is recognized quickly and a quality electrolyte solution is used, OES should be considered the treatment of choice for treating diarrhea in calves. They can effectively increase plasma volume, correct moderate metabolic acidemia and electrolyte abnormalities, and increase blood glucose concentrations, all of which were seen in this study. In severely dehydrated calves, combining OES with hypertonic saline at $4 \mathrm{~mL} /$ $\mathrm{kg}$ can increase the speed and efficiency of increasing plasma volume compared with OES alone. However, the practice of treating calves with 1 to $2 \mathrm{~L}$ of LRS given IV or SC as the sole treatment for diarrhea is not recommended.

\section{CONCLUSIONS}

Oral electrolyte products remain the gold standard for resuscitating calves with diarrhea and are effective in rapidly correcting mild to moderate dehydration and acidemia. Small infusions of IV fluids can be used but are most effective when combined with OES. In severely dehydrated calves, hypertonic saline can be combined with OES to improve efficacy. However, in calves with severe acidemia, intravenous bicarbonate solutions are indicated. Subcutaneous fluids were absorbed very slowly in this study and do not appear to be effective for rapid resuscitation of calves with diarrhea. Used alone, they likely represent a poor treatment option and should only be used for supportive therapy following the initial correction of hypovolemia and metabolic acidosis.

\section{ACKNOWLEDGMENTS}

This study was supported by Boehringer Ingelheim Vetmedica Inc. (Duluth, GA). The authors acknowledge Hannah Sylvester (North Carolina State University) for technical support.

\section{REFERENCES}

Aydogdu, U., R. Yildiz, H. Guzelbektes, A. Naseri, E. Akyuz, and I. Sen. 2018. Effect of combinations of intravenous small-volume hypertonic saline chloride, acetate Ringer, sodium bicarbonate, and 
lactate Ringer solutions along with oral fluid on the treatment of calf diarrhea. Pol. J. Vet. Sci. 21:273-280.

Bachmann, L., B. Schmidt, U. Rauwolf, J. Wenge, and M. Coenen. 2012. Change of plasma volume, osmolality, and acid-base status in healthy calves after feeding of milk and water- and milk-based oral rehydration solutions. J. Dairy Sci. 95:6006-6014.

Berchtold, J. 2009. Treatment of calf diarrhea: Intravenous fluid therapy. Vet. Clin. North Am. Food Anim. Pract. 25:73-99.

Binder, H. J., I. Brown, B. S. Ramakrishna, and G. P. Young. 2014 Oral rehydration therapy in the second decade on the twenty-first century. Curr. Gastroenterol. Rep. 16:376.

Constable, P. D., H. M. Gohar, D. E. Morin, and J. C. Thurmon. 1996. Use of hypertonic-saline dextran solution to resuscitate hypovolemic calves with diarrhea. Am. J. Vet. Res. 57:97-104.

Constable, P. D., W. Grunberg, and L. Carstensen. 2009. Comparative effects of two oral rehydration solutions on milk clotting, abomasal luminal $\mathrm{pH}$, and abomasal emptying rate in suckling calves. J. Dairy Sci. 92:296-312.

Cooley, C. M., J. M. Quimby, S. M. Caney, and L. G. Sieberg. 2018. Survey of owner subcutaneous fluid practices in cates with chronic kidney disease. J. Feline Med. Surg. 20:884-890.

Flores, R. V., C. A. Souza, N. M. Ocarino, V. A. Gheller, M. J. Lopes, M. S. Palhares, and R. Serakides. 2006. Hypertonic and isotonic saline solutions in dehydration therapy in neonate calves: comparison of clinical profile and serum and urinary concentrations of electrolytes. Comp. Clin. Pathol. 15:131-142.

Gaucheron, F. 2005. The minerals of milk. Reprod. Nutr. Dev. 45:473483.

Goodell, G. M., J. Campbell, L. Hoejvang-Nielsen, W. Stansen, and P. D. Constable. 2012. An alkalinizing oral rehydration solution containing lecithin-coated citrus fiber is superior to a nonalkalinizing solution in treating 360 calves with naturally acquired diarrhea. J. Dairy Sci. 95:6677-6686.

Grove-White, D. H., and D. G. White. 1993. Diagnosis and treatment of metabolic acidosis in calves: A field study. Vet. Rec. 133:499501.

Hartling, L., S. Bellemare, N. Wiebe, K. Russell, T. P. Klassen, and W. Craig. 2006. Oral versus intravenous rehydration for treating dehydration due to gastroenteritis in children. Cochrane Database Syst. Rev. 2006:CD004390.

Kasari, T. R., and J. M. Naylor. 1985. Clinical evaluation of sodium bicarbonate, sodium L-lactate, and sodium acetate for the treatment of acidosis in diarrheic calves. J. Am. Vet. Med. Assoc. 187:392-397

Leal, M. L., S. S. Fialho, F. C. Cyrillo, H. G. Bertagnon, E. L. Ortolani, and F. J. Benesi. 2012. Intravenous hypertonic saline solution $(7.5 \%)$ and oral electrolytes to treat of calves with noninfectious diarrhea and metabolic acidosis. J. Vet. Intern. Med. 26:1042-1050.

Naylor, J. M. 1987. Severity and nature of acidosis in diarrheic calves over and under one week of age. Can. Vet. J. 28:168-173.

Reiten, M., T. Rousing, P. T. Thomsen, N. D. Otten, B. Forkman, H. House, J. T. Sørensen, and M. K. Kirchner. 2018. Mortality, diarrhea and respiratory disease in Danish dairy heifer calves: Effect of production system and season. Prev. Vet. Med. 155:21-26.

Roush, G. C., R. Kaur, and M. E. Ernst. 2014. Diuretics: A review and update. J. Cardiovasc. Pharmacol. Ther. 19:5-13.

Smith, G. W. 2009. Treatment of calf diarrhea: Oral fluid therapy. Vet. Clin. North Am. Food Anim. Pract. 25:55-72.
Smith, G. W. 2017. Myth: One oral electrolyte is just as good as another. Pages 41-47 in Proc. Am. Assoc. Bovine Pract., Omaha, NE. Am. Assoc. Bovine Pract., Ashland, $\mathrm{OH}$.

Smith, G. W., and J. Berchtold. 2014. Fluid therapy in calves. Vet. Clin. North Am. Food Anim. Pract. 30:409-427.

Smith, M. R., and R. J. Greer. 2016. Pilot study on the effect of subcutaneous administration of lactated Ringer's solution on biochemistry parameters in healthy euvolemic cats. Vet. Med. Sci. 2:47-53.

Trefz, F. M., P. D. Constable, and I. Lorenz. 2015. Quantitative physicochemical analysis of acid-base balance and clinical utility of anion gap and strong ion gap in 806 neonatal calves with diarrhea. J. Vet. Intern. Med. 29:678-687.

Trefz, F. M., P. D. Constable, C. Sauter-Louis, A. Lorch, G. KnubbenSchweizer, and I. Lorenz. 2013b. Hyperkalemia in neonatal calves depends on the degree of dehydration and the cause of the metabolic acidosis but does not require the presence of acidemia. J. Dairy Sci. 96:7234-7244.

Trefz, F. M., M. Feist, and I. Lorenz. 2016. Hypoglycaemia in hospitalised neonatal calves: Prevalence, associated conditions and impact on prognosis. Vet. J. 217:103-108.

Trefz, F. M., A. Lorch, M. Feist, C. Sauter-Louis, and I. Lorenz. 2012. Construction and validate of a decision tree for treating metabolic acidosis in calves with neonatal diarrhea. BMC Vet. Res. 8:238.

Trefz, F. M., A. Lorch, M. Feist, C. Sauter-Louis, and I. Lorenz. 2013a. The prevalence and clinical relevance of of hyperkalemia in calves with neonatal diarrhea. Vet. J. 195:350-356.

Trefz, F. M., and I. Lorenz. 2017. Plasma potassium concentrations in neonatal diarrhoeic calves are correlated with serum aldosterone concentrations but not with insulin concentrations. Vet. J. 230:41-44

Trefz, F. M., I. Lorenz, A. Lorch, and P. D. Constable. 2017. Clinical signs, profound acidemia, hypoglycemia, and hypernatremia are predictive of mortality in 1,400 critically ill neonatal calves with diarrhea. PLoS One 12:e0182938.

Urie, N. J., J. E. Lombard, C. B. Shiveley, C. A. Kopral, A. E. Adams, T. J. Earleywine, J. D. Olson, and F. B. Garry. 2018. Preweaned heifer management on US dairy operations: Part 1. Descriptive characteristics of preweaned heifer raising practices. J. Dairy Sci. 101:9168-9184

Walker, P. G., P. D. Constable, D. E. Morin, J. F. Drackley, J. H Foreman, and J. C. Thurmon. 1998a. A reliable, practical, and economical protocol for inducing diarrhea and severe dehydration in the neonatal calf. Can. J. Vet. Res. 62:205-213.

Walker, P. G., P. D. Constable, D. E. Morin, J. F. Foreman, J. F. Drackley, and J. C. Thurmon. 1998b. Comparison of hypertonic saline-dextran solution and lactated Ringer's solution for resuscitating severely dehydrated calves with diarrhea. J. Am. Vet. Med. Assoc. 213:113-121.

\section{ORCIDS}

V. Doré $\odot$ https://orcid.org/0000-0002-1564-7312

D. M. Foster (ㄴ) https://orcid.org/0000-0002-6859-9801

G. W. Smith () https://orcid.org/0000-0001-5700-8810 